\title{
The Anterior Intraparietal Sulcus Mediates Grasp Execution, Independent of Requirement to Update: New Insights from Transcranial Magnetic Stimulation
}

\author{
Nichola J. Rice, Eugene Tunik, and Scott T. Grafton \\ Department of Psychological and Brain Sciences, Center for Cognitive Neuroscience, Dartmouth College, Hanover, New Hampshire 03755
}

\begin{abstract}
Although a role of the intraparietal sulcus (IPS) in grasping is becoming evident, the specific contribution of regions within the IPS remains undefined. In this vein, transcranial magnetic stimulation (TMS) was delivered to the anterior (aIPS), middle (mIPS), and caudal (cIPS) IPS in two tasks designed to dissociate the potential roles of the IPS in either grasp planning or execution (task 1) and its involvement in error detection or error correction (task 2). Determining the involvement of specific regions of the IPS in perceptual (planning and error detection) versus motor (execution and correction) components of grasping allowed us to assess the ecological validity of competing computational models attempting to simulate reach-to-grasp movements. In task 1, we demonstrate that, when no on-line adjustment is necessary, TMS to aIPS (but not mIPS or cIPS) disrupts grasping; this disruption is only elicited when TMS is applied during the execution (but not the planning) phase of the movement. Task 2 reveals that TMS to aIPS (but not mIPS or cIPS) disrupts grasping in the presence of a perturbation; this disruption is only elicited when TMS is applied during the error correction (but not error detection) phase of the movement. We propose that the specific contribution of the aIPS in grasping is in the on-line computation of a difference vector based on motor goal, efference copy, and sensory inputs. This computation is performed for both stable and perturbed motor goals.
\end{abstract}

Key words: motor control; grasping; intraparietal sulcus; dorsal stream; computational models; perception and action

\section{Introduction}

Our everyday interactions with objects are characterized by smooth reach-to-grasp actions that can be efficiently modified in the presence of perturbations to the sensorimotor system. Electrophysiological recordings in nonhuman primates (Sakata et al., 1995; Murata et al., 2000) and imaging data from humans (Binkofski et al., 1999; Culham et al., 2003; Frey et al., 2005) have identified a frontoparietal reach-to-grasp network, lesions of which in both humans (Binkofski et al., 1999) and monkeys (Gallese et al., 1994; Fogassi et al., 2001) lead to pronounced deficits in hand preshaping during grasping. The induction of brief virtual lesions through transcranial magnetic stimulation (TMS) offers a unique window in causally attributing anatomy with function to understand the specific role of nodes within this network in goal-directed reach-to-grasp actions.

A recent study has demonstrated that TMS to the anterior portion of the intraparietal sulcus (aIPS) disrupts goaldependent on-line adjustments of grasp (Tunik et al., 2005) when compared with movements for which no explicit update is required; these deficits were elicited when TMS was delivered

Received April 17, 2006; revised July 3, 2006; accepted July 5, 2006.

This work was supported by United States Public Health Service Grants NS44393 and NS33505.

Correspondence should be addressed to Scott T. Grafton, Department of Psychological and Brain Sciences, Center for Cognitive Neuroscience, HB 6162 Moore Hall, Dartmouth College, Hanover, NH 03755. E-mail: scott.t.grafton@dartmouth.edu.

DOI:10.1523/JNEUROSCI.1641-06.2006

Copyright $\odot 2006$ Society for Neuroscience $\quad$ 0270-6474/06/268176-07\$15.00/0 within $65 \mathrm{~ms}$ after the perturbation of the object, leading the authors to suggest a role of aIPS in error detection. A slightly more posterior region within the IPS has also been implicated in on-line control. TMS to this portion of the sulcus disrupts online corrective responses when reaching toward perturbed targets (Desmurget et al., 1999) and grasping perturbed objects (Glover et al., 2005).

Although the involvement of the IPS in on-line control is becoming well established in both reaching (Desmurget et al., 1999; Pisella et al., 2000; Grea et al., 2002) and grasping (Glover et al., 2005; Tunik et al., 2005), its specific contribution to the computations used in on-line control is not understood. Because this region is implicated in grasping in stable contexts (Binkofski et al., 1999; Culham et al., 2003; Frey et al., 2005) and for representing the goal of an intended action (Hamilton and Grafton, 2006), there is strong reason to attribute a broader role for IPS than the planning of hand configurations. Although the abovementioned TMS studies attribute a causal relationship between the IPS and on-line control, the increase in complexity from the noperturbation to perturbation condition may have made the IPS more susceptible to TMS-induced deficits. Additionally, because visual feedback was continuously provided, these studies could not dissociate a role of IPS in error detection from correction.

The present study probes the specific involvement of the IPS in the control of prehension by using TMS to disrupt the anterior (aIPS), middle (mIPS), and caudal (cIPS) aspects of the IPS during grasping. Specifically, we ask whether these regions play a role 
in (1) generating versus executing a reach-to-grasp movement performed in a stable context, and (2) detecting a mismatch between the intended action and a new context versus correcting the adaptive response. This is the first attempt to map the precise roles of regions along the IPS in grasping behavior. In addition, we assess the ecological validity of competing computational models simulating reach-to-grasp movements.

\section{Materials and Methods}

Subjects

Ten healthy subjects participated in the study after providing written informed consent (six females, four males; mean \pm SD age, $24.9 \pm 4.83$ years old). Dartmouth Institutional Review Board approval was granted for all procedures. All subjects were right handed, as determined using the Edinburgh Handedness Inventory (Oldfield, 1971).

\section{Procedure}

Subjects were seated at a table with their chin positioned in a chin rest to minimize head movement. Each trial began with subjects placing their thumb and index finger on a start button directly in front of them. Subjects were required to grasp a rectangular object $(8 \times 2.5 \times 1 \mathrm{~cm})$ that was mounted on the shaft of a motor (model S6MH4; Kollmorgen, Brattleboro, VT), positioned at shoulder level; the position of the motor from the subject was adjusted to ensure that each subject could comfortably reach the target $(57 \mathrm{~cm}$ from the edge of the table). The object was to be grasped using a precision grip, with their index finger and thumb oriented along the vertical dimension. Two different trial types were tested: trials in which the motor rotated at the start of movement to perturb the orientation of the object (perturbation task), and trials in which the motor kept the object motionless (no-perturbation task). In both tasks, visual feedback was controlled by liquid crystal shutter glasses (Plato System; Translucent Technologies, Toronto, Ontario, Canada), which remained opaque between each trial. The opening of the shutter glasses always served as the "Go" cue. The two tasks are detailed below.

No-perturbation task. The shutter glasses were programmed to open for $200 \mathrm{~ms}$ at the start of each trial. During this viewing period, subjects received visual feedback of the starting orientation of the object, which had an equal probability of being either horizontal (grasp the small dimension) or vertical (grasp the large dimension). The shutter glasses then closed for the remainder of the trial. On a given trial, TMS was delivered (1) during the viewing period, i.e., during the planning/detection phase (TMS 1), (2) at start button release, signaled by release of the start button, i.e., execution phase (TMS 2), or (3) not at all (no TMS) (Fig. 1a). The no-TMS trials were included to control for interblock fatigue or practice effects. In the TMS conditions, the first pulse was delivered simultaneous with the opening of the shutter glasses (TMS 1) or hand-button release (TMS 2), the second pulse was delivered $100 \mathrm{~ms}$ after the first.

Perturbation task. The viewing period was the same as in the noperturbation task. After closure of the shutter glasses, the motor reoriented the object by $90^{\circ}$, which was not perceived by the subjects. The release of the start button, at the onset of the reach-to-grasp movement, triggered the reopening of the shutter glasses for $200 \mathrm{~ms}$, allowing subjects to view the perturbed object orientation. Thus, a horizontal-tovertical perturbation necessitated a small-to-large adjustment in grasp aperture, whereas a vertical-to-horizontal perturbation necessitated a large-to-small adjustment in aperture. On a given trial, TMS was delivered (1) during the second viewing period, i.e., during the error detection phase (TMS 1), (2) immediately after the second viewing period, i.e., during the error correction phase (TMS 2), or (3) not at all (no TMS) (Fig. 1b). The no-TMS trials were included to control for interblock fatigue or practice effects. In the TMS conditions, the first pulse was delivered simultaneously with the opening of the shutter glasses (TMS 1) or closing of the shutter glasses (TMS 2), and the second pulse was delivered $100 \mathrm{~ms}$ after the first.

The experiment consisted of six blocks of 60 trials. Within each block, there was an equal number of perturbation and no-perturbation trials, presented randomly. This ensured that subjects were forced to make a movement plan during the early viewing phase of each trial because, in the no-perturbation trials, they would not receive any additional infor- a
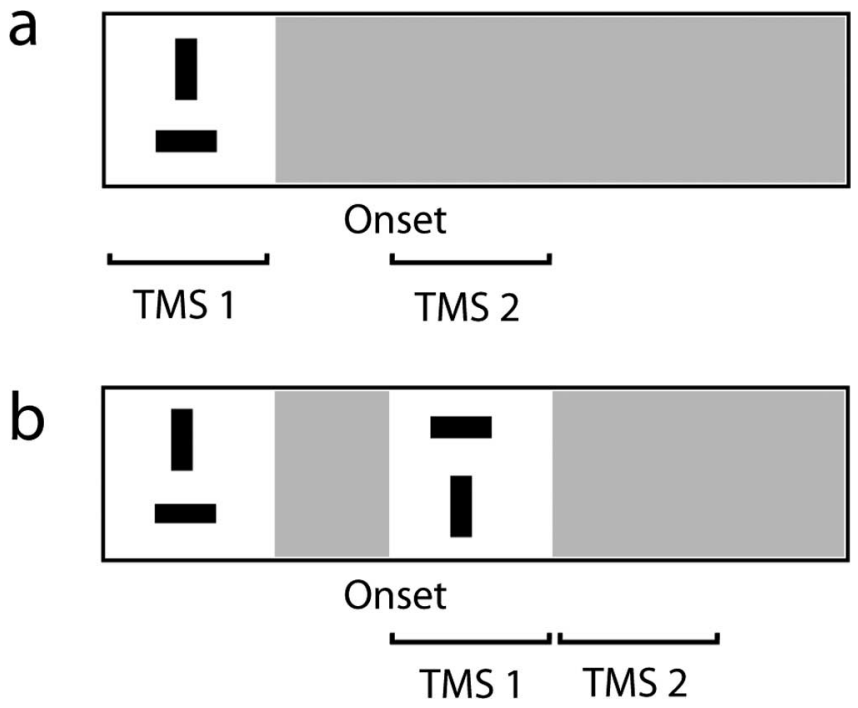

Figure 1. The timing of experimental events in the no-perturbation task $(\boldsymbol{a})$ and the perturbation task $(\boldsymbol{b})$. Filled gray boxes indicate the time when the shutter glasses are closed; white boxes indicate the time that the shutter glasses are open. The black rectangle represents the target, which is presented vertically (large grasp) or horizontally (small grasp).

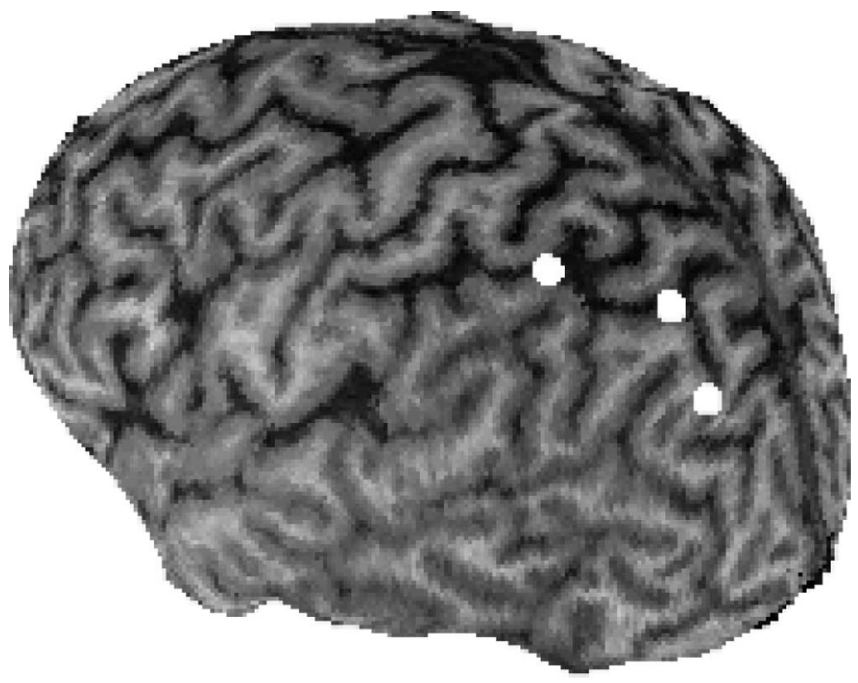

Figure 2. A three-dimensional rendering of one subject's structural MRI. The white dots indicate three cortical sites chosen for stimulation: the most anterior site representing alPS, the middle site representing mIPS, and the caudal site representing cIPS.

mation (whereas in the perturbation task, subjects were then required to update this initial plan using visual feedback provided during the second viewing phase).

\section{Localization of brain sites and TMS}

Three cortical sites in the left hemisphere were chosen for stimulation: (1) the most anterior region of the IPS (aIPS), located at the junction between the anterior extent of the IPS and the inferior postcentral sulcus (Culham et al., 2003; Frey et al., 2005); (2) a region located near the apex of the IPS, near the middle of the sulcus (mIPS); and (3) a region in the most caudal part of the IPS (cIPS) (Fig. 2). The cortical sites for each subject are depicted in supplemental Figure 1 (available at www.jneurosci. org as supplemental material). The proximity of the three regions robustly controlled for the spatial specificity of TMS-induced effects. TMS was delivered to each of the sites on a block-by-block basis, with two blocks per brain site to control for fatigue effects. The order of the blocks was counterbalanced across subjects. Ear plugs were provided to dampen 
the noise associated with the discharge from the TMS coil as well as the rotation of the motor.

A high-resolution three-dimensional volumetric structural magnetic resonance image (MRI) was obtained for each subject (3 $\mathrm{T}$ magnetic resonance imaging scanner; Philips, Aachen, Germany), and the cortical surface was displayed as a three-dimensional representation using Brainsight Frameless Stereotaxic software (Rogue Research, Montreal, Quebec, Canada). Each targeted cortical site was demarcated on the threedimensional image using the same software. The position of the coil and the subject's head were monitored using a Polaris Optical Tracking System (Northern Digital, Waterloo, Ontario, Canada). Positional data for both rigid bodies were registered in real time to a common frame of reference and were superimposed onto the reconstructed threedimensional MRI of the subject using the Brainsight software (Rogue Research). Thus, the center of the coil (stimulation locus) was continuously monitored to be over the site of interest. For all sites, the TMS coil was held tangential to the surface of the skull, with the handle pointing downward. An adjustable frame was used to hold the TMS coil firmly in place. The coil was held to the subject's skull by the experimenter using their left hand, and the right hand was used to stabilize the head to the coil. Head movements were restricted by the use of a chin rest, and the position of the coil to the head was monitored continuously on-line using Brainsight (Rogue Research). In a previous TMS investigation (Schenk et al., 2005), head movements during a reach-to-grasp task were monitored and shown to be negligible under such conditions.

A Neotonus (Marietta, GA) PNS stimulator (model N-0233-A-110V) with an air-cooled iron-core butterfly-shaped coil was used to administer TMS. Pulse duration for this stimulator and head coil is $180 \mu$ s (at 100\% of operating power). Double-pulse TMS (interstimulus interval, $100 \mathrm{~ms}$ ) was applied at $110 \%$ of motor threshold. Motor threshold was determined as the intensity required to produce a visible contraction of the intrinsic hand muscles $50 \%$ of the time with the coil positioned over the hand area of the left primary motor cortex.

Previous TMS studies investigating the role of aIPS in grasping have shown that a single pulse of TMS to aIPS produces a delay of, on average, $88 \mathrm{~ms}$ in time of peak aperture and $146 \mathrm{~ms}$ increase in movement time (Tunik et al., 2005). This provides an indication of the behavioral effects of a single pulse of TMS to aIPS on this sort of task. As such, it would be anticipated that a single pulse of TMS during either the initial viewing period (i.e., planning phase) or the second viewing period in the perturbation task (i.e., error detection phase) would not be enough to disrupt the perceptual phase (given that the goggles were open for $200 \mathrm{~ms}$ ). We intended for the double-pulse TMS, with an interstimulus interval of 100 $\mathrm{ms}$, to disrupt aIPS during the entire perceptual time windows used in this study. It is important to note that the double-pulse TMS applied in this study must be distinguished from other investigations using pairedpulse TMS, in which the two TMS pulses are typically spaced between 1 and $30 \mathrm{~ms}$ apart (Kujirai et al., 1993; Oliveri et al., 2000; Koch et al., 2006). It has been shown in these studies that, at specific interstimulus intervals, the second TMS pulse can abolish the effects of the first; for example, Oliveri et al. (2000) have shown that the second TMS pulse abolishes the effects of the first when paired-pulse TMS is applied to posterior parietal cortex but only with an interstimulus interval of $5 \mathrm{~ms}$ (significantly shorter than our interstimulus interval).

After completing the experiment, all participants were required to complete a side-effects questionnaire, as recommended by Machii et al. (2006). Five subjects reported neck pain, which they all attributed to sitting with their head in a chin rest. Four subjects reported headache, two subjects reported scalp pain, and one subject reported difficulty concentrating as a result of TMS stimulation. All of these side effects were rated as being mild.

\section{Analysis and statistics}

Kinematic data were obtained by localizing the three-dimensional position of infrared light-emitting diodes (sampling rate, $100 \mathrm{~Hz}$; Optotrak 3020; Northern Digital) attached to the tip of the right index finger, thumb, and the first metacarpophalangeal joint (MPJ). Off-line, missing samples were interpolated and the data were filtered at $10 \mathrm{~Hz}$ using custom-written Labview (National Instruments, Austin, TX) software.

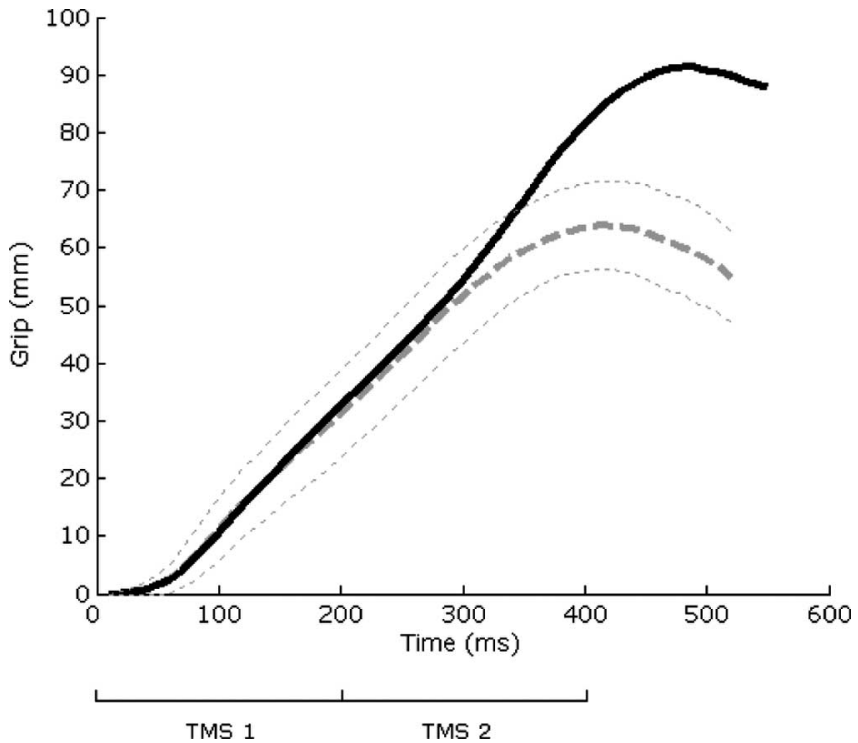

Figure 3. Latency of adaptive response. Group mean aperture trajectory profiles in the noTMS condition for the small-to-large perturbed object condition (thick solid black line) and the small object in the no-perturbation task (thick dashed gray line). Thin dashed gray lines show $\pm 1 S D$ in the small-object condition. The latency of the adaptive response is represented by the time at which the small-to-large grip profile diverges from the small-object grip profile $\pm 1 S D$.

The onset and offset of the movement were defined as the time at which the velocity of the MPJ marker exceeded and then fell below $50 \mathrm{~mm} / \mathrm{s}$, respectively. Trials were excluded from analysis when subjects failed to make a successful grasping movement and when missing data made it impossible to determine the onset or offset of the movement. In the perturbation task, $95.3 \%$ of trials were included in the analysis $(94.3 \%$ of aIPS trials, $96.7 \%$ of cIPS trials, and $95.7 \%$ of mIPS trials), and, in the no-perturbation task, $96 \%$ of trials were included in the analysis (95\% of aIPS trials, $98.7 \%$ of cIPS trials, and $96.3 \%$ of mIPS trials).

To determine that our TMS 1 and TMS 2 pulses were delivered at appropriate times in each task, preliminary analysis was conducted. For the no-perturbation task, average reaction time for all of the no-TMS trials from each block was determined, defined as the time of movement onset. This analysis revealed that subjects initiated their responses after the initial viewing period (mean \pm SD reaction time, $312.31 \pm 62.02 \mathrm{~ms}$ ). This confirms that our planning (TMS 1) and execution (TMS 2) pulses were delivered at the appropriate times (i.e., the execution phase of the movement was not occurring within our initial viewing period and, as such, was not overlapping with the planning phase). For the perturbation task, latency of adaptive response was computed. This was computed by determining the time at which the group mean grip aperture of the no-TMS trials in the perturbation condition (i.e., small-to-large perturbation) diverged from the group mean $\pm 1 \mathrm{SD}$ grip aperture of the no-TMS trials in the no perturbation task (i.e., small); Figure 3 illustrates the time at which this adaptive response occurred (group mean $\pm \mathrm{SD}$, $352 \pm 46.14$ ). This confirms that the implementation of the adaptive response occurred well after the effective TMS 1 epoch but was within the effective TMS 2 epoch. In other words, the pulses in the TMS 1 condition were time locked to the error detection of the perturbation, whereas the pulses in the TMS 2 condition were time locked to the error correction stage of the adaptive response.

Kinematic data were analyzed separately for the transport and grasp components of the movement. Transport-related dependent measures included the following: movement time (MTt), defined by the time interval between movement onset and offset; peak velocity (PVt), defined as the maximum value of the first derivative of the three-dimensional position of the MPJ marker; and time of peak velocity (TPVt), defined as the time interval between peak velocity and movement onset. Grasprelated dependent measures included the following: maximum grip aperture (MGAg), defined as the three-dimensional distance between the 
Table 1. Summary of results for trials in which subjects grasped the large object in the no-perturbation task

\begin{tabular}{|c|c|c|c|c|c|c|c|c|c|}
\hline \multirow[b]{2}{*}{ Variable } & \multicolumn{3}{|l|}{ alPS } & \multicolumn{3}{|l|}{ CIPS } & \multicolumn{3}{|l|}{ mIPS } \\
\hline & TMS 1 & TMS 2 & № TMS & TMS 1 & TMS 2 & № TMS & TMS 1 & TMS 2 & No TMS \\
\hline MTt (ms) & $580.50(68.10)$ & $592.60(70.44)$ & $586.15(64.78)$ & $591.70(79.96)$ & $592.63(68.67)$ & $589.93(74.18)$ & $580.50(62.27)$ & $597.90(52.01)$ & 581.37 (49.16) \\
\hline $\mathrm{PVt}(\mathrm{mm} / \mathrm{s})$ & $1110.44(151.58)$ & $1081.52(111.57)$ & $1083.68(151.69)$ & $1102.31(163.67)$ & $1069.51(150.31)$ & $1059.73(160.49)$ & $1102.54(147.98)$ & $1073.91(129.08)$ & $1079.97(141.21)$ \\
\hline TPVt (ms) & $254.85(48.18)$ & $255.70(48.94)$ & $271.03(34.59)$ & 253.50 (43.71) & $258.33(37.12)$ & $255.73(40.02)$ & 257.01 (42.59) & $269.45(41.40)$ & $262.73(41.90)$ \\
\hline $\operatorname{MGAg}(\mathrm{mm})$ & 115.55 (12.55) & $114.63(12.56)$ & $116.00(12.97)$ & $116.38(10.63)$ & 116.36 (12.43) & $115.97(10.99)$ & $117.53(12.86)$ & $115.53(12.54)$ & $116.70(12.56)$ \\
\hline TMGAg (ms) & $469.30(71.17)$ & $452.45(66.60)$ & $474.10(51.93)$ & $462.00(71.63)$ & $465.08(63.30)$ & $456.93(68.53)$ & $458.23(51.24)$ & $465.65(52.41)$ & 455.67 (41.32) \\
\hline $\mathrm{PVg}(\mathrm{mm} / \mathrm{s})$ & 563.64 (153.37) & $639.83(190.77)$ & $563.14(188.10)$ & $596.26(186.41)$ & $578.93(213.89)$ & $587.47(188.88)$ & $579.11(153.09)$ & $586.31(164.03)$ & 578.85 (157.42) \\
\hline
\end{tabular}

Table reports group mean and SDs (shown in parentheses) for each dependent measure in the no-perturbation task.

Table 2. Summary of results for trials in which the subjects grasped the target that perturbed from small to large in the perturbation task

\begin{tabular}{|c|c|c|c|c|c|c|c|c|c|}
\hline \multirow[b]{2}{*}{ Variable } & \multicolumn{3}{|l|}{ aIPS } & \multicolumn{3}{|l|}{ CIPS } & \multicolumn{3}{|l|}{ mIPS } \\
\hline & TMS 1 & TMS 2 & No TMS & TMS 1 & TMS 2 & No TMS & TMS 1 & TMS 2 & No TMS \\
\hline MTt (ms) & $602.19(59.30)$ & $612.15(62.73)$ & 614.58 (57.47) & $610.53(57.36)$ & $615.00(63.63)$ & $606.03(63.56)$ & $605.93(49.52)$ & $608.05(54.43)$ & 608.73 (49.81) \\
\hline $\mathrm{PVt}(\mathrm{mm} / \mathrm{s})$ & 1062.43 (110.08) & 1070.14 (127.46) & 1055.62 (120.63) & 1067.82 (147.07) & 1058.89 (137.18) & $1083.88(151.92)$ & $1069.02(132.44)$ & $1073.86(132.40)$ & 1049.90 (139.97) \\
\hline TPVt (ms) & 265.18 (44.96) & $259.63(33.42)$ & $266.51(36.41)$ & $258.48(44.61)$ & $257.90(62.81)$ & 251.53 (49.42) & $264.50(48.44)$ & $264.03(49.66)$ & $269.70(45.66)$ \\
\hline $\operatorname{MGAg}(\mathrm{mm})$ & 112.17 (12.67) & $116.11(12.59)$ & 111.73 (12.04) & $113.32(11.29)$ & $113.54(11.80)$ & $113.17(12.76)$ & 113.62 (13.76) & 114.25 (13.84) & 114.09 (13.38) \\
\hline TMGAg (ms) & $484.61(64.72)$ & 498.18 (40.72) & $492.96(58.87)$ & $492.35(56.47)$ & $490.70(59.65)$ & $482.63(62.55)$ & $484.83(47.01)$ & 488.25 (44.39) & 487.55 (44.47) \\
\hline $\mathrm{PVg}(\mathrm{mm} / \mathrm{s})$ & 561.07 (161.02) & $616.26(176.96)$ & $542.34(142.50)$ & 573.61 (182.79) & 555.59 (137.39) & $562.86(155.46)$ & $581.41(185.63)$ & $593.11(146.57)$ & $578.66(156.45)$ \\
\hline
\end{tabular}

Table reports group mean and SDs (shown in parentheses) for each dependent measure in the perturbation task.

index and thumb markers; time of MGA (TMGAg), defined as the time interval between MGA and movement onset; and peak velocity of grip aperture $(\mathrm{PVg})$, defined as the maximum value of the first derivative of grip aperture.

Empirical evidence suggests that adaptive corrections for small-tolarge perturbations become detectable, on average, $329.8 \mathrm{~ms}$ after movement onset (Paulignan et al., 1991) or at $40 \%$ of movement time (Glover et al., 2005), falling within the epoch of our TMS 2 condition in the perturbation experiment. Conversely, large-to-small adjustments become detectable much later, at $\sim 60 \%$ of MT (Glover et al., 2005), well after the time window during which we delivered TMS to disrupt error correction processes. For this reason, only the small-to-large trial types could be analyzed in the perturbation task. Despite this, including both small-to-large and large-to-small perturbations in the testing session added a dimension of complexity on each trial that was not present in the study of Tunik et al. (2005). To ensure equal power of analysis across experiments, only the trials in which the subjects grasped the large object (i.e., vertically orientated object) were analyzed in the no-perturbation task. Data for the no-perturbation and perturbation tasks was analyzed using separate $3 \times 3$ repeated-measures ANOVA for each dependent measure, with factors TMS site (aIPS, mIPS, and cIPS) and TMS time (TMS 1, TMS 2, and no TMS). For both tasks, preplanned $t$ tests were used for subsequent analysis; this involved comparing TMS 1 and TMS 2 conditions with the no-TMS condition for each TMS site. A significance threshold of 0.05 was adopted. For conciseness, only significant findings are discussed, but data for all variables are presented in Tables 1 (noperturbation task) and 2 (perturbation task).

\section{Results}

No-perturbation task

The no-perturbation task was conducted to investigate the role of the IPS in planning (TMS 1) versus executing (TMS 2) a goaloriented reach-to-grasp action. A significant brain site $\times$ TMS time interaction was observed for $\operatorname{TMGAg}\left(F_{(4,36)}=3.178 ; p=\right.$ $0.025)$ and $\operatorname{PVg}\left(F_{(4,36)}=2.909 ; p=0.035\right)$. Subsequent preplanned $t$ tests were conducted to determine the source of these interactions. Figure $4 a$ illustrates that an earlier TMGAg $(t=$ $-2.283 ; p=0.048$ ) was observed when TMS was applied to aIPS during the execution phase (i.e., TMS 2), relative to the control condition (i.e., no TMS); $p>0.198$ for all other comparisons. Figure $4 b$ illustrates that an increase in $\operatorname{PVg}(t=1.942 ; p=0.084)$ was observed when TMS was applied to aIPS during the execu- a

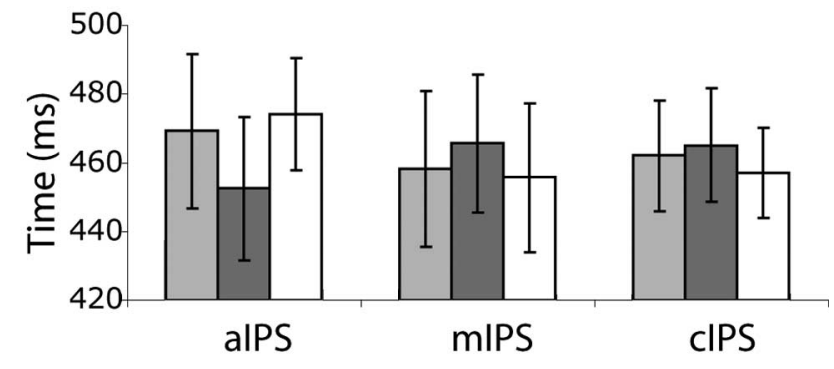

$b$

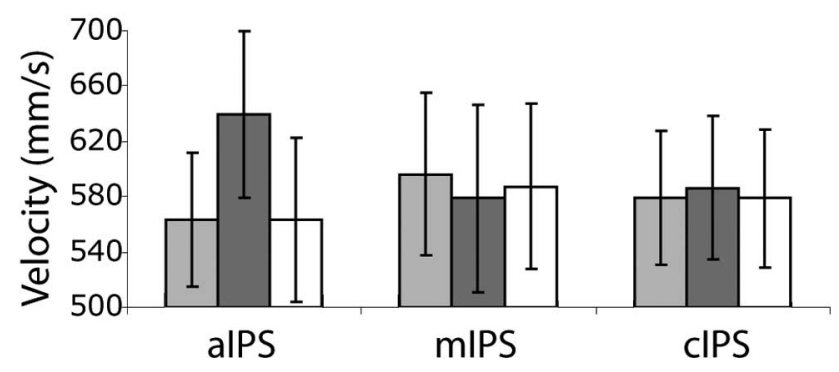

Figure 4. Group mean and SE for TMGAg $(\boldsymbol{a})$ and $\mathrm{PVg}(\boldsymbol{b})$ in the no-perturbation task. Light gray bars represent TMS 1, dark gray bars represent TMS 2, and white bars represent no TMS.

tion phase (i.e., TMS 2), relative to the control condition (i.e., no TMS); $p>0.531$ for all other comparisons. Conversely, no significant interactions were noted for the transport component, although a significant main effect of TMS time was observed for $\operatorname{PVt}\left(F_{(2,18)}=4.551 ; p=0.025\right)$. The overall increase in handtransport velocity was observed in the TMS 1 condition. However, this effect was consistent across brain sites and therefore unlikely accounts for the interactions noted in the grasp-related measures. Overall, these findings suggest a disruption in the grasp (but not the transport) component induced by TMS to aIPS (but not control sites); this disruption was only elicited when TMS was delivered during the execution phase (TMS 2) of the movement. 
a

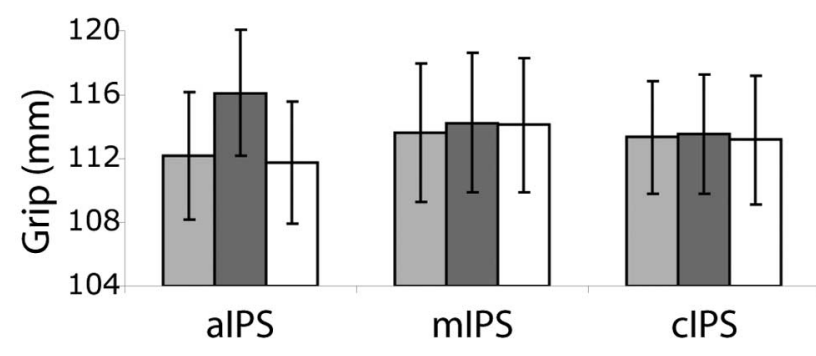

b

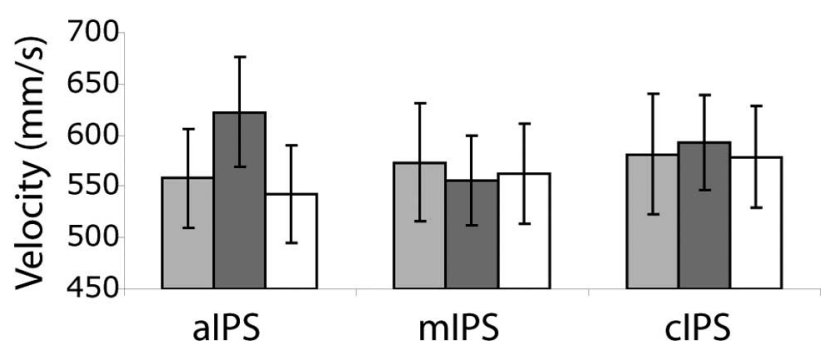

Figure 5. Group mean and SE for $\operatorname{MGAg}(\boldsymbol{a})$ and $\mathrm{PVg}(\boldsymbol{b})$ in the perturbation task. Light gray bars represent TMS 1, dark gray bars represent TMS 2, and white bars represent no TMS.

\section{Perturbation task}

Whereas the no-perturbation task investigated the control of reach-to-grasp actions in stable contexts, the perturbation task probed cortical involvement in on-line updating. Specifically, this task was designed to differentiate roles in detecting a need to adapt a grasp (TMS 1) from the implementation of the corrective response (TMS 2). A significant brain site $\times$ TMS time interaction was noted for $\operatorname{MGAg}\left(F_{(4,36)}=3.897 ; p=0.010\right)$ and $\mathrm{PVg}$ $\left(F_{(4,36)}=2.905 ; p=0.037\right)$. Subsequent preplanned $t$ tests were conducted to determine the source of these interactions. Figure $5 a$ illustrates that an increase in $\operatorname{MGAg}(t=3.713 ; p=0.005)$ was observed when TMS was applied to aIPS during the corrective phase (i.e., TMS 2) of the movement, relative to the control condition (i.e., no TMS); $p>0.424$ for all other comparisons. Figure $5 b$ illustrates that an increase in PVg $(t=2.998 ; p=0.017)$ was observed when TMS was applied to aIPS during the corrective phase (i.e., TMS 2) of the movement, relative to the control condition (i.e., no TMS); $p>0.299$ for all other comparisons. A significant main effect of TMS time was observed for MGAg $\left(F_{(2,18)}=4.224 ; p=0.031\right)$, with an overall increase in MGAg in the TMS 2 condition; this effect was consistent across brain sites and therefore unlikely accounts for the interactions noted in the grasp-related measures. No significant main effects or interactions were noted in the reach (transport) kinematics. These findings suggest that a disruption in the grasp (but not the transport) component was induced by TMS applied to aIPS but not control sites, but only when TMS was applied during the error correction phase (TMS 2) of the movement.

\section{Discussion}

Using TMS, we sought to investigate the specific role the anterior, middle, and caudal aspects of the IPS in prehension. Subjects were required to reach and grasp an object in a stable context (no-perturbation task) or under conditions in which an adaptive response was required (perturbation task). TMS was applied at specific time intervals in each task to map the role of these regions in (1) generating and executing a grasping movement performed in a stable context, and (2) updating the ongoing movement by detecting a mismatch between the intended action and context and correcting the action. In the no-perturbation task, it was shown that TMS to aIPS (but not mIPS or cIPS) disrupted grasping when applied during the execution phase of the movement. In the perturbation task, it was shown that TMS to aIPS (but not mIPS or cIPS) disrupted grasping when applied during the correction phase of the movement. Our findings support previous studies suggesting that aIPS is involved in grasping (Binkofski et al., 1999; Culham et al., 2003; Frey et al., 2005; Tunik et al., 2005). However, this study provides us with important new insights regarding the specific role of this region in grasping.

This is the first study to provide evidence of a functional role of aIPS in grasping when there is no explicit requirement to update the movement. Moreover, the temporal specificity of the TMS-induced deficit was time locked to the execution of the movement. In addition, the present data replicate previous findings that aIPS is involved in on-line updating (Tunik et al., 2005), but we extend these findings by localizing the role of aIPS to one of error correction. It is likely that the results from our noperturbation and perturbation tasks reflect a common role of aIPS in on-line control. That is, we contend that aIPS is critically involved in the integration of sensory and motor components for orchestrating an action, regardless of an explicit need to update the movement. Such a proposal has important implications for neural networks modeling reach-to-grasp movements.

Hoff and Arbib (1993) proposed a model that uses sensory information about the limb and target (e.g., distance and size) to estimate the movement time of the transport and grasp before the movement is initiated. Independent transport and grasp controllers are then activated simultaneously during execution. This model allows for modification of the transport and grasp components, in response to perturbations, through feedback mechanisms. Ulloa and Bullock (2003) proposed a model that represents the limb and target as vectors. A difference vector is computed by determining the difference between the target vector and the current position vector. A common gating signal multiplies both components of the difference vector to specify movement speed. The system responds to perturbations by recomputing the difference vector. Both of these models simulate a system that can respond on-line to perturbations of either the transport or grasp component. Additionally, both models account for synchronous ending of the transport and grasp components of the movement.

A critical difference between these models is in the premovement phase. In the Hoff and Arbib (1993) model, sensory information is used to estimate movement time of the transport and grasp components before the movement is initiated. Although there might be other brain areas that perform this sort of estimate, our results suggest that this is not calculated within the IPS. Ulloa and Bullock (2003) eliminate the need for such a planning phase by continuous updating of the difference vector. The present findings, by localizing TMS-induced deficits to the execution stages of the movement, support the Ulloa and Bullock model. It has been proposed that the gating signal for updating the difference vector is generated by the basal ganglia, the current state of the sensorimotor system is represented by the hand area of primary motor cortex, and the difference vector may be computed in the posterior parietal cortex (Bullock and Grossberg, 1988; Ulloa and Bullock, 2003). Given that the basal ganglia send projections to the anterior intraparietal (AIP) area in monkeys (Clower et al., 2005), these two regions may form a functional network involved in on-line control. Our findings support this 
thesis and indicate a critical role of aIPS in the computation of a difference vector.

Another finding, consistent with the Ulloa and Bullock (2003) model, is that TMS to aIPS produced deficits in the grasp but not the transport component. This model would predict that a perturbation in either the transport or grasp component of the movement would effect only the respective component. Paired with recent functional imaging evidence demonstrating that aIPS represents the goals of grasping actions (Hamilton and Grafton, 2006), the computations performed by aIPS seem to be restricted to the action/solution pertaining to the goal (in our case, grasp aperture). This hypothesis is strongly supported by the finding that TMS to aIPS can induce deficits in either grasp aperture or hand-forearm orientation, depending on the goal of the task (Tunik et al., 2005). As such, we would predict that, if we had used a perturbation in the transport component, we would expect to observe TMS-induced deficits in transport but not grasp.

Previous TMS investigations have suggested that the IPS is only required when explicit on-line adjustments are required for both grasping (Glover et al., 2005; Tunik et al., 2005) and reaching (Desmurget et al., 1999). A possible explanation for the difference between the results of the present study and previous investigations is that, previously, the increase in complexity from the no-perturbation to the perturbation condition may have made the IPS more susceptible to TMS-induced deficits. Moreover, because the no-perturbation condition served as the control condition in these studies, deficits in this condition could not be analyzed. The present study overcame this limitation by analyzing the no-perturbation condition independently. An additional element of control and complexity was added by controlling the precise timing of the visual feedback and by randomly interleaving the perturbation and no-perturbation trials. These design issues allow us to make a compelling case that aIPS is involved in grasping when there is no explicit requirement to update the movement. This thesis is supported by neuroimaging (Culham et al., 2003; Frey et al., 2005) and patient (Binkofski et al., 1999) studies suggesting that aIPS is involved in grasping when there is no explicit requirement to update the ongoing movement. However, the data expand on such studies by localizing the involvement of aIPS to one of grasp execution.

One might argue that, in the no perturbation task, subjects initiated and executed their responses differently than in the perturbation, because subjects may have gained some information concerning trial type based on the timing of the TMS pulses (i.e., if TMS was delivered during the first viewing phase, the trial was always a no-perturbation trial). We feel this is unlikely. In the kinematic data presented in Table 1, there are no significant differences between the early TMS condition and no-TMS condition in any of these variables, for any of the TMS sites. In addition, the presence of a significant interaction that is specific to the late TMS condition, and only so for the aIPS site, is indicative of the high degree of specificity for our effects.

This study opens several interesting directions to pursue. First, if the IPS is involved in executing and correcting a grasping action, which regions of the frontoparietal reach-to-grasp network are involved in planning the grasp and detecting changes in the sensorimotor context that would necessitate an adaptive response? A possible candidate may be the ventral premotor cortex $(\mathrm{PMv})$. This thesis is validated by electrophysiological recordings in nonhuman primates showing that neurons in this region code specific actions, respond to visual stimuli (including graspable stimuli), and respond during action observation (Rizzolatti et al., 2002). Furthermore, PMv is known to share connections with
AIP (Rizzolatti et al., 2002), which we have demonstrated is critically involved in execution and updating of goal-directed grasping. Finally, a recent TMS study (Davare et al., 2006) has shown that repetitive stimulation of PMv early in the movement disrupts grasping, suggesting that it may be involved in grasp preparation. However, the availability of visual feedback and the fact that TMS was only delivered after the Go signal in that study made it impossible to dissociate planning from execution.

In conclusion, the present experiment was designed to map the specific role of regions along the IPS in prehension. By applying TMS to various regions along the IPS as subjects were required to perform a grasping movement, it was shown that the area within the IPS responsible for grasping behavior can be localized to the aIPS. More importantly, this study has shown that aIPS is involved in executing a grasp when there is no requirement to update the ongoing movement, and, in the presence of an explicit perturbation, it is also involved in executing the correction. Finally, we contend that this computation may involve the continuous updating of a difference vector, based on efference copy of the motor command and sensory input.

\section{References}

Binkofski F, Buccino G, Stephan KM, Rizzolatti G, Seitz RJ, Freund HJ (1999) A parieto-premotor network for object manipulation: evidence from neuroimaging. Exp Brain Res 128:210-213.

Bullock D, Grossberg S (1988) Neural dynamics of planned arm movements: emergent invariants and speed accuracy properties during trajectory formation. Psychol Rev 95:49-90.

Clower DM, Dum RP, Strick PL (2005) Basal ganglia and cerebellar inputs to "AIP." Cereb Cortex 15:913-920.

Culham JC, Danckert SL, DeSouza JFX, Gati JS, Menon RS, Goodale MA (2003) Visually guided grasping produces fMRI activation in dorsal but not ventral stream brain areas. Exp Brain Res 153:180-189.

Davare M, Andres M, Cosnard G, Thonnard J-L, Olivier E (2006) Dissociating the role of ventral and dorsal premotor cortex in precision grasping J Neurosci 26:2260-2268.

Desmurget M, Epstein CM, Turner RS, Prablanc C, Alexander GE, Grafton ST (1999) Role of the posterior parietal cortex in updating reaching movements to a visual target. Nat Neurosci 2:563-567.

Fogassi L, Gallese V, Buccino G, Craighero L, Fadiga L, Rizzolatti G (2001) Cortical mechanism for the visual guidance of hand grasping movements in the monkey: a reversible inactivation study. Brain 124:571-586.

Frey SH, Vinton D, Norlund R, Grafton ST (2005) Cortical topography of human anterior intraparietal cortex active during visually guided grasping. Cognit Brain Res 23:397-405.

Gallese V, Murata A, Kaseda M, Niki N, Sakata H (1994) Deficit of hand preshaping after muscimol injection in monkey parietal cortex. NeuroReport 5:1525-1529.

Glover S, Miall RC, Rushworth MFS (2005) Parietal rTMS disrupts the initiation but not the execution of on-line adjustments to a perturbation of object size. J Cogn Neurosci 17:124-136.

Grea H, Pisella L, Rossetti Y, Desmurget M, Tilikete C, Grafton S, Prablanc C, Vighetto A (2002) A lesion of the posterior parietal cortex disrupts online adjustments during aiming movements. Neuropsychologia 40:2471-2480.

Hamilton AF, Grafton ST (2006) Goal representation in human anterior intraparietal sulcus. J Neurosci 26:1133-1137.

Hoff B, Arbib MA (1993) Models of trajectory formation and temporal interaction of reach and grasp. J Mot Behav 25:175-192.

Koch G, Franca M, Albrecht U-V, Caltagirone C, Rothwell J (2006) Effects of paired pulse TMS of primary somatosensory cortex on perception of a peripheral electrical stimulus. Exp Brain Res 172:416-424.

Kujirai T, Caramia MD, Rothwell JC, Day BL, Thompson PD, Ferbert A, Wroe S, Asselman P, Marsden CD (1993) Corticocortical inhibition in human motor cortex. J Physiol (Lond) 471:501-519.

Machii K, Cohen D, Ramos-Estebanez C, Pascual-Leone A (2006) Safety of rTMS to non-motor cortical areas in healthy participants and patients. Clin Neurophysiol 117:455-471.

Murata A, Gallese V, Luppino G, Kaseda M, Sakata H (2000) Selectivity for 
the shape, size, and orientation of objects for grasping in neurons of monkey parietal area AIP. J Neurophysiol 83:2580-2601.

Oldfield RC (1971) Assessment and analysis of handedness - Edinburgh inventory. Neuropsychologia 9:97-113.

Oliveri M, Caltagirone C, Filippi MM, Traversa R, Cicinelli P, Pasqualetti P, Rossini PM (2000) Paired transcranial magnetic stimulation protocols reveal a pattern of inhibition and facilitation in the human parietal cortex. J Physiol (Lond) 529:461-468.

Paulignan Y, Jeannerod M, Mackenzie C, Marteniuk R (1991) Selective perturbation of visual input during prehension movements. 2. The effects of changing object size. Exp Brain Res 87:407-420.

Pisella L, Grea H, Tilikete C, Vighetto A, Desmurget M, Rode G, Boisson D, Rossetti Y (2000) An "automatic pilot" for the hand in human posterior parietal cortex: toward reinterpreting optic ataxia. Nat Neurosci 3:729-736.
Rizzolatti G, Fogassi L, Gallese V (2002) Motor and cognitive functions of the ventral premotor cortex. Curr Opin Neurobiol 12:149-154.

Sakata H, Taira M, Murata A, Mine S (1995) Neural mechanisms of visual guidance of hand action in the parietal cortex of the monkey. Cereb Cortex 5:429-438.

Schenk T, Ellison A, Rice N, Milner AD (2005) The role of V5/MT+ in the control of catching movements: an rTMS study. Neuropsychologia 43:189-198.

Tunik E, Frey SH, Grafton ST (2005) Virtual lesions of the anterior intraparietal area disrupt goal-dependent on-line adjustments of grasp. Nat Neurosci 8:505-511.

Ulloa A, Bullock D (2003) A neural network simulating human reach-grasp coordination by continuous updating of vector positioning commands. Neural Netw 16:1141-1160. 